\title{
Accurate Quantification of Small-Diameter Tubular Structures in Isotropic CT Volume Data Based on Multiscale Line Filter Responses
}

\author{
Yoshinobu Sato $^{1}$, Shuji Yamamoto ${ }^{2}$, and Shinichi Tamura ${ }^{1}$ \\ 1 Division of Interdisciplinary Image Analysis \\ Osaka University Graduate School of Medicine, Japan \\ yoshi@image.med.osaka-u.ac.jp, http://www.image.med.osaka-u.ac.jp/yoshi \\ 2 Department of Radiology, Osaka University Hospital, Japan
}

\begin{abstract}
A method fully utilizing multiscale line filter responses is presented to estimate the point spread function (PSF) of a CT scanner and diameters of small tubular structures based on the PSF. The estimation problem is formulated as a least square fitting of a sequence of multiscale responses obtained at each medial axis point to the precomputed multiscale response curve for the ideal line model. The method was validated through phantom experiments and demonstrated to accurately measure small-diameter structures which are significantly overestimated by conventional methods based on the full width half maximum (FWHM) and zero-crossing edge detection.
\end{abstract}

\section{Introduction}

Diameter measurement of tubular structures in 3D medical data is an essential tool for quantitative evaluations of blood vessels, bronchial airways, and other similar anatomical structures. A typical method is to extract medial axes of the structures and then quantitate the width of the contour of cross-section orthogonal to the axis [1, 2, 3]. The diameters of small-diameter structures are known to be overestimated when they are measured based on the cross-sectional contours 3]. There are inherent limits on the accuracy of diameter measurement due to finite resolution of imaging scanners [4] and blurring involved in edge detectors. The influences of these limits are also discussed on thickness measurement of thin sheet structures [5], [6], [7]. In order to overcome these limits, Reinhardt et al. 8] incorporated the effects of the point spread function (PSF) of an imaging scanner into a measurement procedure for two diameters of the cross-sectional pattern specific to bronchial airways based on model fitting of one-dimensional original intensity profiles. Its main drawbacks, however, are as follows.

- The PSF width is assumed to be known.

- The direct use of the original intensity profiles is sensitive to noise.

In this paper, we propose a method for measurement of small-diameter tubular structures based on model fitting of multiscale Gaussian blurred second derivatives along intensity ridges. The proposed method has the following advantages.

C. Barillot, D.R. Haynor, and P. Hellier (Eds.): MICCAI 2004, LNCS 3216, pp. 508-515 2004.

(C) Springer-Verlag Berlin Heidelberg 2004 
- The PSF width is estimated in the method, and thus any pre-calibration process is unnecessary.

- The use of Gaussian blurred responses along intensity ridges is expected to be relatively insensitive to noise.

- Scale-dependent constraints can be incorporated into a measurement procedure so as to improve the measurement accuracy and stability.

In our current formulation, it is assumed that 3D data sets are acquired from a CT scanner whose PSF is approximated by an isotropic Gaussian function and the cross section of tubular structure is approximated by a pill-box function (although either of these assumptions can be potentially removed). We compare the proposed method with the full width half maximum (FWHM) measure and diameter measurement based on zero-crossing contours of cross-sections.

\section{Method}

\subsection{Modeling Multiscale Line Filter Response of Ideal 3D Line}

A 3D tubular structure (line) orthogonal to the $x y$-plane is modeled as

$$
\operatorname{Line}(\boldsymbol{x} ; D)=\operatorname{Pill-Box}(x, y ; D),
$$

where $\boldsymbol{x}=(x, y, z)^{\top}, D$ is the line diameter, and

$$
\operatorname{Pill-Box}(x, y ; D)=\left\{\begin{array}{l}
1 \sqrt{x^{2}+y^{2}}<\frac{D}{2} \\
0 \sqrt{x^{2}+y^{2}}>\frac{D}{2}
\end{array} .\right.
$$

Here, line structures are assumed to be brighter than surrounding regions.

The point spread function (PSF) of a CT scanner is assumed to be described by $\operatorname{Gauss}\left(\boldsymbol{x} ; \sigma_{p s f}\right)$, where $\operatorname{Gauss}(\boldsymbol{x} ; \sigma)$ is the isotropic 3D Gaussian function, and $\sigma_{p s f}$ denotes the PSF width. The intensity function of $3 \mathrm{D}$ data, $f_{\text {line }}\left(\boldsymbol{x} ; D, \sigma_{p s f}\right)$, of line diameter $D$ imaged by a CT scanner of PSF width $\sigma_{p s f}$ is given by

$$
f_{\text {line }}\left(\boldsymbol{x} ; D, \sigma_{p s f}\right)=\operatorname{Line}(\boldsymbol{x} ; D) * \operatorname{Gauss}\left(\boldsymbol{x} ; \sigma_{p s f}\right),
$$

where $*$ denotes the convolution operation.

When the Gaussian filters are applied to the acquired 3D data by postprocessing, the Gaussian filtered 3D data of a line are described by

$$
f_{\text {line }}\left(\boldsymbol{x} ; D, \sigma_{p s f}, \sigma_{f}\right)=\operatorname{Line}(\boldsymbol{x} ; D) * \operatorname{Gauss}\left(\boldsymbol{x} ; \sigma_{p s f}\right) * \operatorname{Gauss}\left(\boldsymbol{x} ; \sigma_{f}\right),
$$

where $\sigma_{f}$ is the filter width. The Gaussian filtered line with intensity height $H$ is described by $H \cdot f_{\text {line }}\left(\boldsymbol{x} ; D, \sigma_{p s f}, \sigma_{f}\right)$. In our problem, $D, H$, and $\sigma_{p s f}$ are unknown and need to be estimated from $3 \mathrm{D}$ data while $\sigma_{f}$ is known.

In the proposed approach, we fully utilize the scale-dependency of the line filter responses in order to estimate line diameter $D$ as well as PSF width $\sigma_{p s f}$ 
and line height $H$. We consider the line filter responses, $R(\sigma ; D)$, of the ideal 3D line at the line axis, that is, $\boldsymbol{x}=(0,0, z)$, which is given by

$$
R(\sigma ; D)=\sigma^{2} \cdot \frac{\partial^{2}}{\partial x^{2}} f_{\text {line }}\left(0,0, z ; D, \sigma_{p s f}, \sigma_{f}\right),
$$

where $\sigma=\sqrt{\sigma_{p s f}^{2}+\sigma_{f}^{2}}$. As shown in the previous work [9], $\sigma^{2}$ is multiplied for scale-normalization of the responses. Given $D, \sigma_{p s f}$, and $\sigma_{f}, R(\sigma ; D)$ can be calculated using Eq. (5). Fig. 1 shows an example of $R(\sigma ; D)$ calculated numerical simulation.

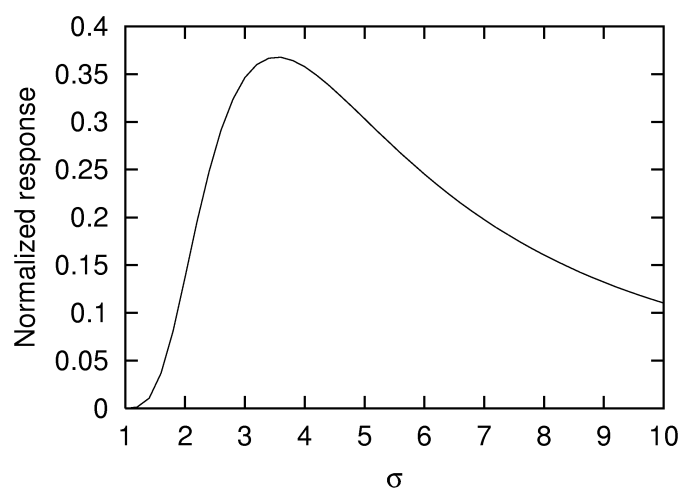

Fig. 1. Normalized line filter responses, $R(\sigma ; D)$, of ideal 3D line at line axis. This plot shows $R(\sigma ; D)$ for $D=10$. Normalized line filter responses have the maximum when $\sigma=0.36 D$ for line structures with a pill-box cross-section.

\subsection{Extracting a Sequence of Multiscale Line Filter Responses on Medial Axis from 3D Data}

A method for localizing the medial axis element [3] is summarized, and then a procedure for extracting a sequence of multiscale filter responses on each medial axis element is described. Hereafter, we call the axis element "axel". It should be noted that the axels are not grouped and only fragmentally detected at each voxel.

Let the intensity function of Gaussian filtered 3D data be $f\left(\boldsymbol{x} ; \sigma_{p s f}, \sigma_{f}\right)$ or $f(\boldsymbol{x} ; \sigma)$, where $\sigma=\sqrt{\sigma_{p s f}^{2}+\sigma_{f}^{2}}$. The gradient vector of $f(\boldsymbol{x} ; \sigma)$ is defined as $\nabla f(\boldsymbol{x} ; \sigma)=\left(f_{x}(\boldsymbol{x} ; \sigma), f_{y}(\boldsymbol{x} ; \sigma), f_{z}(\boldsymbol{x} ; \sigma)\right)^{\top}$, where partial derivatives of $f(\boldsymbol{x} ; \sigma)$ are represented as $f_{x}(\boldsymbol{x} ; \sigma)=\frac{\partial}{\partial x} f(\boldsymbol{x} ; \sigma)$, and so on. The Hessian matrix of $f(\boldsymbol{x} ; \sigma)$ is given by

$$
\nabla^{2} f(\boldsymbol{x} ; \sigma)=\left[\begin{array}{lll}
f_{x x}(\boldsymbol{x} ; \sigma) & f_{x y}(\boldsymbol{x} ; \sigma) & f_{x z}(\boldsymbol{x} ; \sigma) \\
f_{y x}(\boldsymbol{x} ; \sigma) & f_{y y}(\boldsymbol{x} ; \sigma) & f_{y z}(\boldsymbol{x} ; \sigma) \\
f_{z x}(\boldsymbol{x} ; \sigma) & f_{z y}(\boldsymbol{x} ; \sigma) & f_{z z}(\boldsymbol{x} ; \sigma)
\end{array}\right]
$$


where partial second derivatives of $f(\boldsymbol{x} ; \sigma)$ are represented as $f_{x x}(\boldsymbol{x} ; \sigma)=$ $\frac{\partial^{2}}{\partial x^{2}} f(\boldsymbol{x} ; \sigma), f_{y z}(\boldsymbol{x} ; \sigma)=\frac{\partial^{2}}{\partial y \partial z} f(\boldsymbol{x} ; \sigma)$, and so on.

Let the eigenvalues of $\nabla^{2} f(\boldsymbol{x} ; \sigma)$ be $\lambda_{1}, \lambda_{2}, \lambda_{3}\left(\lambda_{1} \geq \lambda_{2} \geq \lambda_{3}\right)$ and their corresponding eigenvectors be $\boldsymbol{e}_{1}, \boldsymbol{e}_{2}, \boldsymbol{e}_{3}\left(\left|\boldsymbol{e}_{1}\right|=\left|\boldsymbol{e}_{2}\right|=\left|\boldsymbol{e}_{3}\right|=1\right)$, respectively. $\boldsymbol{e}_{1}$ is expected to give the tangential direction of the line and the line filter response is obtained as the combination of $\lambda_{2}$ and $\lambda_{3}$ [9], directional second derivatives orthogonal to $\boldsymbol{e}_{1}$.

We assume that the tangential direction of line is given by $\boldsymbol{e}_{1}$ at the voxel around the medial axis. The 2-D intensity function, $c(\boldsymbol{u})\left(\boldsymbol{u}=(u, v)^{\top}\right)$, on the cross-sectional plane of $f(\boldsymbol{x} ; \sigma)$ orthogonal to $\boldsymbol{e}_{1}$, should have its peak on the medial axis. The second-order local approximation of $c(\boldsymbol{u})$ is given by

$$
c(\boldsymbol{u})=f\left(\boldsymbol{x}_{0} ; \sigma\right)+\boldsymbol{u}^{\top} \nabla c_{0}+\frac{1}{2} \boldsymbol{u}^{\top} \nabla^{2} c_{0} \boldsymbol{u},
$$

where $u \boldsymbol{e}_{2}+v \boldsymbol{e}_{3}=\boldsymbol{x}-\boldsymbol{x}_{0}, \nabla c_{0}=\left(\nabla f \cdot \boldsymbol{e}_{2}, \nabla f \cdot \boldsymbol{e}_{3}\right)^{\top}(\nabla f$ is the gradient vector, that is, $\left.\nabla f\left(\boldsymbol{x}_{0} ; \sigma\right)\right)$, and

$$
\nabla^{2} c_{0}=\left[\begin{array}{cc}
\lambda_{2} & 0 \\
0 & \lambda_{3}
\end{array}\right]
$$

$c(\boldsymbol{u})$ should have its peak on the medial axis of the line. The peak is located at the position satisfying

$$
\frac{\partial}{\partial u} c(\boldsymbol{u})=0 \quad \text { and } \quad \frac{\partial}{\partial v} c(\boldsymbol{u})=0
$$

By solving Eq. (9), we have the offset vector, $\boldsymbol{p}=\left(p_{x}, p_{y}, p_{z}\right)^{\top}$, of the peak position from $\boldsymbol{x}_{0}$ given by $\boldsymbol{p}=s \boldsymbol{e}_{2}+t \boldsymbol{e}_{3}$, where $s=-\frac{\nabla f \cdot \boldsymbol{e}_{2}}{\lambda_{2}}$ and $t=-\frac{\nabla f \cdot \boldsymbol{e}_{3}}{\lambda_{3}}$. For the medial axis to exist at the voxel $\boldsymbol{x}_{0}$, the peak of $c(\boldsymbol{u})$ needs to be located in the territory of voxel $\boldsymbol{x}_{0}$. Thus, the medial axis element, that is, medial axel, is detected only if $\left|p_{x}\right| \leq 0.5 \&\left|p_{y}\right| \leq 0.5 \&\left|p_{z}\right| \leq 0.5$ (voxels). By combining the voxel position $\boldsymbol{x}_{0}$ and offset vector $\boldsymbol{p}$, the medial axel is localized at subvoxel resolution.

We define the line filter response as $\sigma_{f}^{2} \sqrt{\lambda_{2} \lambda_{3}}$ in this paper, where $\sigma_{f}^{2}$ is multiplied to normalize the responses (although additional normalization will be incorporated in the later section when the PSF is considered). A sequence of multiscale line filter responses, $R_{i}$, of different filter widths $\sigma_{f_{i}}(i=1,2,3, \ldots)$ is extracted at each detected medial axel as follows.

1. The medial axel detection and line filtering are performed at different scales $\sigma_{f_{i}}(i=1,2,3, \ldots, n)$.

2. The medial axel having the strongest line filter response is selected among all the detected axels irrespective to scales. Let the strongest axel be found at $\boldsymbol{x}_{0}$ of $\sigma_{f_{i_{0}}}$.

3. The axels nearest and within one-voxel distance to position $\boldsymbol{x}_{0}$ are extracted at adjacent scales $\sigma_{f_{i_{0}+1}}$ and $\sigma_{f_{i_{0}-1}}$. Similarly, the axels are extracted in larger and smaller scales until no axels are found.

4. The above three steps are repeated for the axels to be extracted until all the axels are completed. 


\subsection{Estimation Processes}

The basic strategy is to find $D, H$, and $\sigma_{p s f}$ minimizing the square sum of differences between the model $H \cdot R(\sigma ; D)$ (more precisely, $H \cdot R\left(\sqrt{\sigma_{p s f}^{2}+\sigma_{f}^{2}} ; D\right)$ ) and the data set at each axel, that is, a sequence of multiscale line filter responses, $R_{i}$, of different filter widths $\sigma_{f_{i}}\left(i=i_{s}, \ldots i_{e}\right)$ where $i_{s}$ and $i_{\ell}$ are the smallest and largest scales, respectively, in the sequence at the axel of interest.

The estimation processes consist of two stages. During the first stage, $\sigma_{p s f}$ and $H$ are estimated using the axels which have relatively large diameters and whose positions are close to the voxel center. During the second stage, $D$ is estimated for every sequence of multiscale filter responses using $\sigma_{p s f}$ and $H$ estimated in the first stage.

Estimating PSF of CT imaging and intensity height of line. To accurately estimate PSF width $\sigma_{p s f}$ and intensity height $H, R_{i}$ needs to be fit to the model $R(\sigma ; D)$ in a wide range of scale $\sigma$ including the range around maximum values of $R(\sigma ; D)$. Furthermore, the axel position localized in subvoxel accuracy should be close to the voxel center to guarantee the response is the peak response or close to it. We selected the sequences satisfying the following conditions.

1. $i_{t}<\arg \max _{i} R_{i}$, where $\sigma_{f_{i}}$ should be sufficiently large (at least twice as large as the minimum filter scale $\left.\sigma_{f_{1}}\right)$.

2. $\sqrt{\left|p_{x}\right|^{2}+\left|p_{y}\right|^{2}+\left|p_{z}\right|^{2}}<d_{t}$, where $d_{t}$ is a constant representing the maximum distance to the voxel center to be allowed. In the experiment, we used $d_{t}=0.3$ (voxels).

For the sequences of multiscale filter responses satisfying the above conditions, $\sigma_{p s f}, D$, and $H$ are searched which minimize

$$
E_{1}\left(\sigma_{p s f}, H, D\right)=\sum_{i=i_{s}}^{i_{\ell}}\left\{H \cdot R\left(\sqrt{\sigma_{p s f}^{2}+\sigma_{f_{i}}^{2}} ; D\right)-\frac{\sigma_{p s f}^{2}+\sigma_{f_{i}}^{2}}{\sigma_{f_{i}}^{2}} R_{i}\right\}^{2},
$$

where $i_{s}$ and $i_{\ell}$ are the smallest and largest scales in the extracted sequence of multiscale filter responses at the axel of interest.

In the experiments, exhaustive search was applied to $D$ and $\sigma_{p s f} . D$ was discretized from 0.02 to 24.0 voxels with 0.02 voxel interval, and $\sigma_{p s f}$ was discretized from 0.02 to 4.0 voxel with 0.02 voxel interval. For all the combinations of discretized $D$ and $\sigma_{p s f}, H$ was estimated by minimizing Eq. (10). With known $D$ and $\sigma_{p s f}$, the estimation of $H$ minimizing Eq. (10) is formulated as a linear square problem. Thus, $D, \sigma_{p s f}$, and $H$ are obtained for each sequence of multiscale responses. We assume that $\sigma_{p s f}$ and $H$ are not locally variable. $\sigma_{p s f}$ and $H$ are obtained as averages of all the results from the sequences of multiscale responses considered at this stage. 
Diameter estimation. For all the extracted sequences of multiscale filter responses, using known $H$ and $\sigma_{p s f}, D$ is searched minimizing

$$
E_{2}(D)=\sum_{i=i_{s}}^{i_{\ell}}\left\{H \cdot R(\sigma ; D)-\frac{\sigma^{2}}{\sigma_{f_{i}}^{2}} R_{i}\right\}^{2},
$$

where $\sigma=\sqrt{\sigma_{p s f}^{2}+\sigma_{f_{i}}^{2}}$. In the experiments, exhaustive search was applied to $D$. $D$ was discretized from 0.02 to 24.0 voxels with 0.02 voxel interval,

\section{$3 \quad$ Results}

CT volume data of several diameters of acrylic rods were acquired using a Toshiba Aquilion CT scanner. Isotropic voxel imaging was performed with voxel size of $0.5 \mathrm{~mm}^{3}$. The diameters of acrylic rods were $3.0,1.6,1.0,0.75$, and 0.5 $\mathrm{mm}$. A cross-sectional image in the $y z$-plane of the acquired CT data and its volume rendered image are shown in Fig. 2(a). The rods were straight and the angle of their axes was approximately 20 degrees to the $x y$-plane of the CT coordinate system. The volume data were trimmed and sinc-interpolated so that the voxel interval was $0.25 \mathrm{~mm}$. The multiscale filter widths were $\sigma_{f_{i}}=2^{\frac{i-1}{4}}$ (voxels), where $i=1,2, \ldots, 11$ (the voxel size is $0.25 \mathrm{~mm}^{3}$ ).

The estimation results in the first stage were $\sigma_{p s f}=1.61$ (voxels) $=0.40$ (mm) and $H=1054$, where the voxel size was that of the interpolated volume data, that is, $0.25 \mathrm{~mm}^{3}$. Fig. 2 (b) shows the results of diameter estimation at the second stage. The averages and standard deviations of the experimentally estimated diameters of five rods are indicated in Fig. 2(b). Since the results of the proposed method were experimentally measured, the averages and standard deviations for diameters $3.0,1.6,1.0,0.75$, and $0.5 \mathrm{~mm}$ are indicated as errorbars. (The standard deviations were very small for diameters 3.0 and 1.6, and thus their error-bars collapsed.) The ideal relation was plotted as a dotted line. Fig. 2(b) also shows simulation results of the diameters estimated using full width half maximum (FWHM) and using zero-crossings of radial directional second derivatives orthogonal to medal axis. These results are plotted as dashed lines since they are numerically computed. In the simulations, $\sigma_{p s f}=0.40(\mathrm{~mm})$ was used. In the simulations of zero-crossings, computation of second derivatives was assumed to be combined with Gaussian blurring of standard deviation of 1.2 $($ voxels $)=0.3(\mathrm{~mm})$. In diameter estimations using FWHM and zero-crossings, significant overestimation was observed when the actual diameter was $1.0 \mathrm{~mm}$ or less. In the proposed diameter estimation, accurate measurement was realized even for diameters $1.0 \mathrm{~mm}, 0.75 \mathrm{~mm}$, and $0.5 \mathrm{~mm}$.

\section{Discussion and Conclusions}

We have described a method for accurate measurement of small-diameter tubular structures. This work provides a unified approach based on multiscale filter 


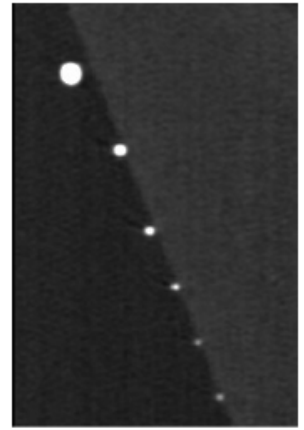

(a)

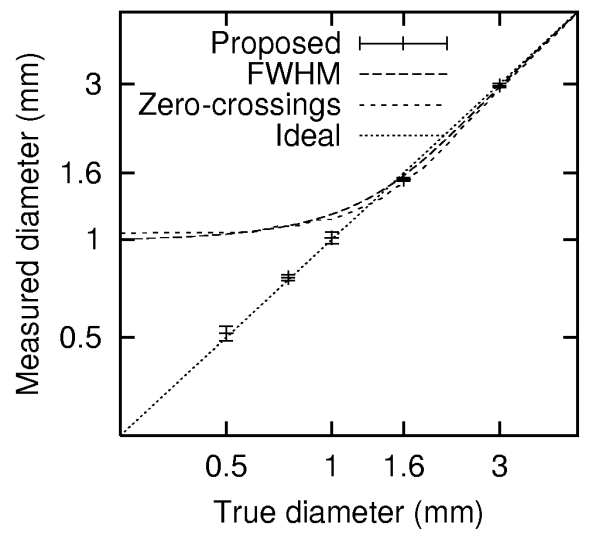

(b)

Fig. 2. Phantom experiments. (a) CT volume data of several diameters of acrylic rods. Cross-sectional image in the $y z$-plane (left) and volume rendered image (right). (b) Results of diameter measurement. Averages and standard deviations of experimentally estimated diameters of five rods using the proposed method are indicated as error-bars. The ideal relation is plotted as a dotted line. Estimated diameters based on full width half maximum (FWHM) and zero-crossing edge detection were numerically computed and are plotted as dashed lines.

responses to the estimation of the point spread function (PSF) of a CT scanner as well as the estimation of diameters. The method is validated through phantom experiments and demonstrated to accurately measure small-diameter structures which are significantly overestimated by conventional methods based on full width half maximum (FWHM) and zero-crossing edge detection.

Because the method is based on second derivatives, it is insensitive to the DC component, that is, the background level, of image intensity. However, inaccuracy in the estimated intensity height $H$ may affect the estimated diameter accuracy (although previous methods based on FWHM and zero-crossings are insensitive to $H)$. We are planning to quantitatively evaluate this effect. Future work will also include the effects of inaccuracy in the PSF width $\sigma_{p s f}$. In this paper, we have assumed isotropic imaging and circular cross-section of the straight tubular structures on a uniform background. The validation needs to be completed on the robustness with respect to anisotropic resolution, non-circular cross-section, the curvature of the structure, and a non-uniform background. (The effects of curvatures on line filter responses were partly evaluated in [9].)

The proposed method is related to the heuristics that the scale at which the maximum normalized response is obtained is selected as the optimal scale of the tubular structures [9],[10],[11]. The optimal scale $\sigma_{o p t}$ is regarded as the true radius, that is, $\sigma_{o p t}=0.5 D$, when cross-sectional intensity distributions are Gaussian [9], while the relation $\sigma_{o p t}=0.36 D$ is observed when they have pill-box shapes (as shown in Fig. 1). In this heuristics, the effect of the imaging scanner PSF is not considered. When the effect of PSF is considered, the maximum 
response is inherently not obtainable for small-diameter structures which do not satisfy the condition $\frac{1}{0.36} \sigma_{p s f}<D$. Further, the output of this heuristics is discrete. In the proposed method, by least square fitting of multiscale responses to the theoretical multiscale response curve, accurate, continuous estimates are obtained even for small-diameter structures not satisfying $\frac{1}{0.36} \sigma_{p s f}<D$.

For clinical application, a multiscale tracking method for tubular structures in 3D data as described in 11. can be effectively combined with the proposed method. We are now developing a method for scale-space tracking of extracted axels to combine with the proposed method.

Acknowledgment. The authors would like to thank Dr. H. Shikata for the permission of using CT volume data of the acrylic rod phantom.

\section{References}

1. Frangi AF, Niessen WJ, Hoogeveen RM, et al.: Model-based quantitation of 3-D magnetic resonance angiographic images, IEEE Trans Med Imaging, 18(10):946956, 1999.

2. Wink O, Niessen WJ, Viergever MA: Fast delineation and visualization of vessels in 3-D angiographic images, IEEE Trans Med Imaging, 19(4):337-346, 2000.

3. Sato Y, Tamuras S: Detection and quantification of line and sheet structures in 3-D images, Lecture Notes in Computer Science (Proc. Third International Conference on Medical Image Computing and Computer Assisted Intervention (MICCAI2000), Pittsburgh, Pennsylvania), 1935: 154-165, 2000.

4. Hoogeveen RM, Bakker CJG, Viergever MA: Limits to the accuracy of vessel diameter measurement in MR angiography, JMRI - J Magn Reson Imaging, 8(6):12281235, 1998.

5. Prevrhal S, Engelke K, Kalender WA: Accuracy limits for the determination of cortical width and density: the influence of object size and CT imaging parameters, Phys Med Biol, 44(3):751-764, 1999.

6. Dougherty G and Newman DL: Measurement of thickness and density of thin structures by computed tomography: A simulation study, Med Phys, 25(7):13411348, 1999.

7. Sato Y, Tanaka H, Nishii T, et al.: Limits on the accuracy of 3D thickness measurement in magnetic resonance images — Effects of voxel anisotropy -, IEEE Trans Med Imaging, 22(9):1076-1088, 2003.

8. Reinhardt JM, D'Souza ND, Hoffman EA: Accurate measurement of intrathoracic airways IEEE Trans Med Imaging, 16(6):820-827, 1997.

9. Sato Y, Nakajima S, Shiraga N, et al.: Three dimensional multi-scale line filter for segmentation and visualization of curvilinear structures in medical images, Med Image Anal, 2(2):143-168, 1998.

10. Krissian K, Malandain G, Ayache, N, et al.: Model-based detection of tubular structures in 3D images, Comput Vis Image Und, 80(2):130-171, 2000.

11. Aylward SR, Bullitt E: Initialization, noise, singularities, and scale in height ridge traversal for tubular object centerline extraction, IEEE Trans Med Imaging, 21(2):61-75, 2002. 